Ann. Zootech., I979, 28 (4), 423-430.

\title{
Evolution de la composition corporelle du vison mâle en croissance à partir du sevrage
}

Geneviève CHARLET-LERY, Michèle FISZLEWICZ, Marie-Thérèse MOREL et D. ALLAIN*

Laboratoive de Physiologie de la Nutrition

* Laboratoire des Pelages, Toisons et Fourrures

Centre national de Recherches zootechniques, I.N.R.A., 78350, Jouy-en-Josas (France)

\section{Résumé}

L'évolution de la composition corporelle de 36 visons mâles répartis en 6 lots homogenes a été étudiée par sacrifice et analyse des carcasses aux poids moyens de $732,1003,123$ I, I 535, I 759 et $1838 \mathrm{~g}$. Durant la période d'observation, le poids moyen de matières grasses passe de 72,7 à $659,9 \mathrm{~g}$, celui des matières azotées de 140,5 à 320,0 g et celui des matières minérales de 23,4 $46,8 \mathrm{~g}$. Dès le début des observations, lorsque les visons croissent de $732 \mathrm{~g}$ à $\mathrm{I} 003 \mathrm{~g}$ en $\mathrm{I} 2 \mathrm{jours}$, le dépôt journalier des lipides dépasse celui des protéines $(5,2$ contre $4,3 \mathrm{~g} / \mathrm{j})$, bien que la rétention protéique soit importante $(36 \mathrm{p}$. Ioo de la rétention totale). Pendant les périodes d'observation la rétention journalière des protéines tombe de $4,3 \mathrm{~g} / \mathrm{j}$ à $0,3 \mathrm{~g} / \mathrm{j}$ tandis que celles des dépôts gras s'élève de $5,2 \mathrm{~g} / \mathrm{j}$ à $8,5 \mathrm{~g} / \mathrm{j}$. Les rendements des dépôts énergétique et azoté par rapport aux quantités ingérées correspondantes sont particulièrement faibles : IO, I et 3,3 p. I oo entre 732 et ${ }^{8} 88 \mathrm{~g}$. Le vison se caractérise donc par un dépôt important de matières grasses corporelles qui aboutit à une teneur de 65,5 p. I oo de matière sèche dès l'achèvement de la croissance début novembre et par de très faibles rendements énergétique et azoté que la méthode utilisée a permis de mettre en évidence.

\section{Introduction}

A qualité égale, la classification des peaux de visons est basée sur leur longueur. Aussi l'éleveur s'efforce-t-il d'obtenir des animaux les plus lourds possible, une corrélation élevée existant entre le poids corporel et la taille des peaux (REITEN, 1977). Avant de mettre au point un régime alimentaire rationnel qui satisfasse à ce but, il nous a paru intéressant de connaître les modifications de la composition corporelle de ces animaux au cours de leur croissance. Or, les données bibliographiques fournissent des valeurs de la fixation journalière de l'azote, qui, obtenues 
par collecte des excréta, paraissent nettement excessives lorsqu'on les compare au gain de poids de l'animal, et n'apportent aucun renseignement sur l'importance des dépôts lipidiques.

C'est pourquoi nous avons entrepris l'étude de la variation de la composition corporelle in toto du vison mâle, du sevrage à son poids maximum (début novembre). Les visons ont été sacrifiés à des poids bien définis, étant donné, d'après des études effectuées sur d'autres espèces, que cette composition corporelle dépend davantage du poids que de l'âge.

\section{Matériel et méthodes}

\section{Animaux}

Les observations ont porté sur 36 visons mâles pastel que nous avons répartis en 6 lots homogènes en poids $(750 \mathrm{~g})$ et en âge (70-77 jours) à la mi-juillet. Nous avons tiré au sort le jour même, dans chacun des 6 lots les animaux qui devraient être ultérieurement tués à 750 , I 000 , I 250, I 500, I 750 et $2000 \mathrm{~g}$ ou au début novembre, au cas où ce dernier poids n'aurait pas été atteint à cette date. Les visons ont été pesés tous les 7 jours. Mais quand leur poids approche celui fixé pour le sacrifice, ils sont pesés touts les 2-3 jours; dès que le poids prévu est dépassé de 50 à Ioo $\mathrm{g}$, le vison n'a plus accès à sa nourriture vers I 7 h. Il est sacrifié le lendemain matin vers $9 h$, soit après un jê̂ne d'environ I $6 \mathrm{~h}$, durée suffisante pour assurer une vidange intestinale complète, chez cet animal au transit intestinal rapide (5 heures environ).

\section{Régime alimentaire}

Les visons ont été nourris ad libitum avec un aliment mis sous forme de granulés : par rapport à l'alimentation sous forme de pâtée, ce mode de présentation permet un contrôle plus précis des quantités consommées tout en assurant une croissance identique (Kumeno et al., I970; RoUgeot et al., I97I).

Nous avons utilisé un même aliment (tab1. I) durant l'expérience à forte concentration protéique (44 p. Ioo de la matière sèche (M.S.)) afin d'assurer une croissance élevée à nos animaux (SKREDE, I978). L'apport énergétique est de $5460 \mathrm{kcal} / \mathrm{kgMS}$ et l'énergie métabolisable mesurée sur vison adulte est de $3855 \mathrm{kcal}$. Les quantités ingérées sont contrôlées tous les 7 jours.

\section{Traitement des carcasses}

Après sacrifice par électrocution, l'animal est immédiatement congelé à - $25^{\circ}$. Le cadavre congelé est émincé à l'aide d'un hachoir (type coupe-pain) et passé dans un broyeur "Robot-Coupe S.A. " (capacité $3 \mathrm{~kg}$ ) jusqu'à l'obtention d'un produit homogène. La carcasse broyée est répartie dans des plateaux d'aluminium et congelée pour être lyophilisée. La durée totale de la préparation est courte, la température finale de la masse broyée ne dépasse jamais $5^{\circ} \mathrm{C}$. C'est pour 
TABLEAU $I$

Composition, concentrations énergétique et azotée du régime alimentaive Composition, energy and nitrogen contents of the diet, (p. roo)

Farine de poisson (Fish meal). . . . . . . . . . . . . . . . . . . . . . . . . . . .25

Farine de viande $($ Meat meal). . . . . . . . . . . . . . . . . . . . . . . . . . . . I5

Tourteau de soja 50 (Soybean meal 5o). . . . . . . . . . . . . . . . . . . . . . . . . . . . . . 5

Levure de bière (Brewer yeast) . . . . . . . . . . . . . . . . . . . . . . . . . . . 5

Farine de sang (Blood meal) . . . . . . . . . . . . . . . . . . . . . . . . . . . . 5

Blé toasté (Toasted wheat) . . . . . . . . . . . . . . . . . . . . . . . . . .20,6

Germe de blé $($ Wheat germ) . . . . . . . . . . . . . . . . . . . . . . . . . . . . . 2

Pulpe de betterave deshydratée (Dried beet pulp) . . . . . . . . . . . . . . . . . . . 2

Farine de luzerne (Lucerne meal) . . . . . . . . . . . . . . . . . . . . . . . . . . 2

Suif (Tallow) . . . . . . . . . . . . . . . . . . . . . . . . . . . . . . . . I 2

Saindoux $($ Lavd $)$. . . . . . . . . . . . . . . . . . . . . . . . . . . . . . . . . . 4

Complément minéral (Mineral supplement) $\left(^{*}\right)$. . . . . . . . . . . . . . . . . . . . . . 1,6

Complément vitaminique (Vitamin supplement) $\left(^{* *}\right)$. . . . . . . . . . . . . . . . . . o,8

Protéines brutes (Crude protein) $\mathrm{N} \times 6,25$. . . . . . . . . . . . . . . . . .44 p. Ioo MS

Energie brute (Gross energy) . . . . . . . . . . . . . . . . . . . . .5460 $\mathrm{kcal} / \mathrm{kg} \mathrm{MS}$

(*) En g pour roo kg aliment: carbonate de calcium I 500, sulfate de fer 70 , sulfate de manganèse 10 , carbonate de zinc ro.

(**) Pour $100 \mathrm{~kg}$ aliment : vit : $2500000 \mathrm{UI}$, Vit. D3 : 100.000 UI, Vit. E : $5000 \mathrm{UI}$, vit. Br : $200 \mathrm{mg}$, vit. $\mathrm{B} 2: 600 \mathrm{mg}$, vit. B6:300 mg, vit. PP : $2000 \mathrm{mg}$, acide folique; $50 \mathrm{mg}$, vit Br2; $5 \mathrm{mg}$, Biotine : $2000 \mathrm{mg}$, Pentothénate de calcium : $2000 \mathrm{mg}$, Choline : $100 \mathrm{~g}$, DL méthionine : $300 \mathrm{~g}$.

cette raison que nous n'avons pas utilisé l'azote liquide. La pesée avant congélation et après 1yophilisation permet de calculer la matière sèche de la carcasse La totalité du produit lyophilisé est ensuite homogénéisé dans un broyeur WARINGBLENDOR avant d'être analysé.

- L'azote est dosé sur une prise d'échantillon de $2 \mathrm{~g}$ par la méthode KJELDAHL avec fixation de $\mathrm{NH}_{3}$ par l'acide borique. L'écart entre les doubles n'excède pas I p. Ioo.

- La valeur énergétique se détermine par combustion dans une bombe calorimétrique adiabatique GALIENKAMP et les matières grasses sont calculées par différence en utilisant les coefficients de 5,7 et 9,5. La teneur élevée en lipides même dans les carcasses de $750 \mathrm{~g}(\mathrm{MG}$ p. Ioo MS $=29$ ) ne permet pas de présenter l'échantillon sous forme de pastille nécessaire pour une bonne combustion : nous avons utilisé des petits sacs en polyéthylène comme le préconise NiJkamp (I965) pour le dosage de l'énergie des urines. Le rapport I/3 d'énergie apporté par le plastique et $2 / 3$ par le produit a été aisément respecté. Par contre, cette haute valeur énergétique oblige à limiter les prises d'échantillons à $750 \mathrm{mg}$ maximum ce qui conduit à effectuer les analyses en triple, 1'écart autour de la moyenne n'excédait pas I,5 p. Ioo. Cette erreur doit être imputée à l'échantillonnage et non pas à des difficultés de combustion, toujours correcte lorsque le produit est bien tassé au fond du sac.

- Les matières minérales sont obtenues par calcination à $55^{\circ} \mathrm{C}$ et pendant Io heures de deux échantillons de $50 \mathrm{~g}$. Malgré l'importance de la prise d'essai, l'écart entre ces doubles peut atteindre io p. roo. 


\section{Résultats}

\section{Poids et âge des visons}

Le tableau 2 montre dans quelles conditions a été effectué le plan expérimental. En particulier, la dispersion de poids à l'intérieur des 5 premiers lots est faible avec un C. V. inférieur à $5 \mathrm{p}$. Ioo. Par contre, pour le dernier lot, le poids de 2 ooo $\mathrm{g}$ n'a pu être atteint que pour un seul sujet et, pour une moyenne de poids de I $838 \mathrm{~g}$, la dispersion est nettement plus élevée avec un C. V. de 9 p. Ioo.

Les conditions expérimentales de groupement de poids ont conduit comme il fallait s'y attendre, à une dispersion des âges autour de la moyenne plus élevée que celle des poids.

\section{Composition corporelle (tab1. 2)}

I'étude de chaque lot et la comparaison des lots entre eux permet de dégager les points suivants. De 732 à I $838 \mathrm{~g}$, le poids des matières grasses corporelles passe de 72,7 à $659,9 \mathrm{~g}$, tandis que celui des matières azotées de I40,5 à 320,0 g, c'est-àdire que le poids de la matière grasse est multiplié par 9 tandis que celtui des matières azotées ne fait que doubler. Le rapport matières grasses/matières azotées varie de 0,5 à 2. C'est aux environs de Ioo jours que la quantité de graisses corporelles dépasse celle des protéines. Mais déjà entre les âges de 73 et 88 jours, phase où la croissance atteint $I 8 \mathrm{~g} / \mathrm{j}$, les dépôts journaliers sont déjà en faveur des graisses $(5,2 \mathrm{~g})$ par rapport aux protéines $(4,3 \mathrm{~g})$. Pendant cette courte période, le vison fixe $64,3 \mathrm{~g}$ de protéines soit $36 \mathrm{p}$. Ioo du gain protéique du dernier groupe ( $79,5 \mathrm{~g})$. Mais à la fin de la période d'observation, entre I759 et $I 838 \mathrm{~g}$, les visons ne fixent plus que $0,26 \mathrm{~g} / \mathrm{j}$ de protéines tanđis que le dépôt de matières grasses s'élève à $8,5 \mathrm{~g} / \mathrm{j}$. Cette évolution se fait avec des modifications profondes de la concentration des constituants. La teneur en eau de la carcasse tombe de 66,3 à 45,5 p. Ioo, celle de la matière azotée de 56,9 à $3 \mathrm{I}, 8$ p. Ioo/MS. Par contre, on assiste à une forte augmentation de la teneur en matière grasse qui passe de 29,4 à $65,5 \mathrm{p}$. Ioo/ MS : cette dernière valeur correspond à un pourcentage d'énergie lipidique par rapport à l'énergie totale de 77,5 .

A la fin de 1'essai, on trouve deux fois plus de matières minérales corporelles qu'au début $(23,4 \mathrm{~g}$ contre $46,8 \mathrm{~g} /$ vison), mais leur concentration dans la MS diminue de 9,5 à 4,6 p. Ioo : leur évolution est donc comparable à celle de la matière azotée.

\section{Rendement alimentaire}

Les indices de consommation de chaque lot sont, comme il est normal, de plus en plus élevés à mesure que les animaux sont tués plus tardivement : 4,65 $\mathrm{kg}$ MS 





par $\mathrm{kg}$ de gain de poids vif entre 732 et I $003 \mathrm{~g}$ et I0,93 $\mathrm{kg}$ MS entre 732 et I $838 \mathrm{~g}$ (tabl. 3).

TABLEAU 3

Indice de consommation et efficacité des dépots corporels des 6 groupes de visons selon le poids à l'abattage

Feed conversion ratio and total efficiency of tissue gain according to slaughter weights

\begin{tabular}{|c|c|c|c|c|c|}
\hline Poids vif moyen $(\mathrm{g})$ (Fasted body mean weight $(\mathrm{g})$ ) . & 1003 & $\mathrm{r} 23 \mathrm{I}$ & I 535 & 1759 & 1838 \\
\hline MSI/gain de poids $\mathrm{kg} / \mathrm{kg}$ (Feed/live gain) & 4,65 & 5,83 & $8,5^{2}$ & $9, \mathrm{i} 4$ & Io,93 \\
\hline $\begin{array}{c}\text { Kcal fixées /keal ingérées } \times \text { тоо (Energy gain /energy } \\
\text { intake). }\end{array}$ & $\mathrm{I} 6,3$ & I 5,4 & I I, 9 & I I, 2 & $I O, I$ \\
\hline $\begin{array}{l}\text { Protéines fixées /protéines ingérées } \times \text { Ioo (Protein gain } / \\
\text { protein intake) }\end{array}$ & II, 3 & 6,8 & 4,4 & 4,2 & 3,3 \\
\hline
\end{tabular}

Les efficacités énergétique et protéique des rations prises comme les rapports entre les dépôts énergétique et azoté et les ingérés énergétique et azoté sont faibles quelle que soit la période considérée. Elles atteignent pour le dernier lot observé entre 738 et I $838 \mathrm{~g}$ respectivement Io, I et 3,3 p. Ioo (tabl. 3 ).

\section{Discussion}

On sait que les bilans journaliers d'azote effectués par collecte des excréta sont toujours plus élevés que ceux obtenus par analyse des carcasses même si les premiers ont été effectués de façon continue durant toute la durée de l'expérience. En effet, les nombreuses erreurs inhérentes à la première de ces méthodes concourrent toutes à l'obtention d'une valeur par excès comme l'a montré MaRTiN (Ig66) chez le mouton maintenu en cage à contention. Il est certain que pour le vison laissé libre dans sa cage les erreurs ne peuvent être que relativement plus importantes. Ceci explique que les bilans journaliers d'azote trouvés sur les visons par la majorité des auteurs soient très nettement supérieurs à ceux que permettent de calculer nos observations, jusqu'à 6 fois plus.

Si 1'on considère la courbe de croissance établie par REITEN (I977) on constate que le vison a terminé sa croissance pondérale dès les premiers jours de novembre et que, par la suite, il a tendance à maigrir. On peut donc admettre que le dépôt de graisse que nous avons observé est le dépôt maximal. Le maximum est obtenu très précocement avant la première période de reproduction qui aura lieu en mars, ce qui est très différent de ce que nous observons chez les mammifères domestiques.

Il est donc per1 aisé de comparer l'évolution de la composition corporelle du vison à ces espèces, d'autant plus que la quasi totalité des études ont été effectuées avec des mâles castrés. Par exemple, Mc Graham (I970) montre que chez 
le mouton mâle castrè de 27 mois le poids de graisse est 4 à 5 fois et même jusqu'à Ir fois supérieur à celui des protéines. Par contre, chez des rats mâles âgés de 265 jours nourris ad libitum et pesant $450 \mathrm{~g}$, BEZA et GRASUL (I977) observent un dépôt gras nettement inférieur au dépôt protéique.

Le vison semble donc se singulariser par un dépôt de matière grasse très précoce et une efficacité protéique particulièrement faible.

Ces premiers résultats qui seront complétés par l'étude entre la naissance et le poids de $75^{\circ} \mathrm{g}$ montrent déjà que l'application au vison de la méthode d'analyse des carcasses apporte des précisions qui manquaient jusqu’à présent sur la croissance de cette espèce et sur l'efficacité de ses dépôts corporels. Ils seraient susceptibles d'orienter la recherche de plans d'alimentation plus conformes aux besoins de cet animal.

Accepté pour publication en octobve 1979.

\section{Summary}

\section{Evolution of body composition of growing male mink from weaning}

Body composition was studied in 36 growing male minks that were divided into 6 groups at weaning (in July), on the basis of equal mean body weights ( $750 \mathrm{~g})$ and age (70-77 days). The animals were fed a pelleted diet ad libitum, containing $44 \mathrm{p}$. cent protein and $5460 \mathrm{kcal} / \mathrm{kg} \mathrm{dry}$ matter (table 1 ).

Successive groups were killed at the average weights of 732 , I003, I 23 I, I 535,1759 and I $838 \mathrm{~g}$ (table 2). During the whole experimental period, the mean weight of stored lipids increased from 72.7 to $659.9 \mathrm{~g}$, the protein content from $\mathrm{I} 40.5$ to 320.0 and minerals from 23.4 to $46.8 \mathrm{~g}$. During the first I2 days of the experiment, when the weight of animals increased from 732 to $1003 \mathrm{~g}$, the daily body lipid tetention was higher than for proteins $(5.2 \mathrm{vs} 4.3 \mathrm{~g} / \mathrm{d})$, in spite of the very important protein gain made during this period ( $36 \mathrm{p}$. I oo of the whole experimental period). The daily retention of protein decreased from $4.3 \mathrm{~g} / \mathrm{d}$ to $0.3 \mathrm{~g} / \mathrm{d}$, while the lipid retention increased from 4.2 to $8.5 \mathrm{~g} / \mathrm{d}$ during the experiment. In the growth period $732-\mathrm{I} 838 \mathrm{~g}$, the total energy and protein efficiencies (table 3 ) were very low: I0.I and 3.3 respectively.

It was concluded that mink are characterized by: I) a very high lipid retention resulting at the end of the growth period in a fat content of $65.5 \mathrm{p}$. roo dry matter and 2) very low efficiencies for energy and protein retention revealed by the methods of fasted body analysis used.

\section{Références bibliographiques}

BEza R., Grazur A., I977. Studies on the chemical composition of the rat body during growth and development. Acta. Physiol. Pol., 28, 559-573.

KUMFino F., ITOYAMA K., HASEGAWA J., AOKIS F., I970. Fffect of protein and fat levels in complete pelleted diets on the growth of mink kits. J. anim. Sci., 31, 894-899.

McGraham N., 1970. Growth in sheep. 5 th Symp. on energy metabolism of farm animal. 106-108, A. Schürch and C. Wenk, Ed. Juris Druck +- Verlag Zurich.

MARTIN A. K., I 966 . Some errots in the determination of nitrogen retention of sheep by nitrogen balance studies. Brit. J. Nutr., 20, 325-337.

NiJkAmP H. J., I965. Some remarks about the determination of the heat of combustion value

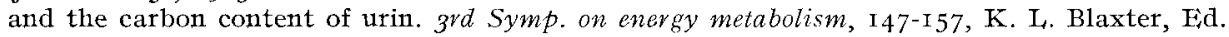
Acad. Press. London New York. 
REITEN J., 1977. Body size and skin size of dark mink. Meld. Norg. Landbrtlogsk., 52, I-I6. Rougeot J., Melcion J.-P., Charleti-Líry G., Delort-Laval J., I97I. Élevage du vison avec un aliment complet aggloméré. Ann. Zootech., 20, 259-262.

SKREDE A., I978. Utilization of fish and animal byproducts in mink nutrition. I. Effect of source and level of protein on nitrogen balance, post weaning growth and characteristics of winter fur quality. Acta Agric. Scand., 28, г05-I 29. 